\title{
Paper
}

\section{Development of Automatic Dust Catcher Robot of Clothes}

\author{
Natsuki NaKamoto* Non-member, $\quad$ Yuhki Kitazono*† ${ }^{* \dagger}$ Member
}

(Received March 12, 2021, revised September 28, 2021)

\begin{abstract}
If you come into contact with others with dust or animal hair on your clothes, there is a high possibility that you will make the other person develop an allergy or not be able to establish good communication. Therefore, it is necessary to remove dusts and more from your clothes in the morning before going to work or school. However, this work is done manually, which is time-consuming and time-consuming. In this paper, we have developed a robot that automatically removes dust from clothes on hangers with a brush. The robot holds and pinches the clothes between two brushes and removes the dust by moving the brushes from top to bottom. It also removes dust from the entire clothes by repeatedly extending the arm with the brush attached and removing the dust. The operation time of this robot is about two minutes. All the user has to do is put the clothes on the hanger, press the start button on the robot, and the robot will remove the dust from the clothes. While the robot is running, the user can spend his time doing other things. For example, by using this robot, you can make better use of your valuable morning time for yourself instead of using it to dust your clothes.
\end{abstract}

Keywords: Clothes, Dust, Brush,

\section{Introduction}

House dust, mites, and animals can cause allergies [1] [4]. It is dangerous to come in contact with allergy sufferers with these substances on your clothes [5] [7]. Therefore, it is necessary to remove dust and other particles from clothes when there is a possibility of contacting with people.

Also, appearance is a quite important point in giving a positive impression on others [8]. According to the questionnaire on marriage activities conducted in 2020 by Partner Agent, a dating agency operated by Tameny Corporation says $48.4 \%$ of men and $65.0 \%$ women of 978 men and women aged 30-39 who have done marriage activities ranked appearance and cleanliness as the most important factor in determining the impression of a partner or the factor that makes a good impression in marriage activities [9]. This is the most common answer for both men and women. In addition, according to the questionnaire conducted in 2019 by this corporation shows, $54.7 \%$ of 320 men and women aged 20-39 answered that cleanliness is an important factor in what they look for in a marriage partner [10].

Thus, wearing clean clothes is one of the things we need to do to maintain good health for ourselves and others, and to build good relationships with others.

In order to wear clean clothes, it is necessary to wash, iron out wrinkles, and remove dust from clothes. In this paper, we focused on the task of removing dust from clothes.

Since the average member of household in Japan is two people, we consider the amount of dust generated at this time [11]. According to "On the generation of dust in

\footnotetext{
$\dagger$ Corresponding:kitazono@kct.ac.jp

* National Institute of Technology, Kitakyushu College
}

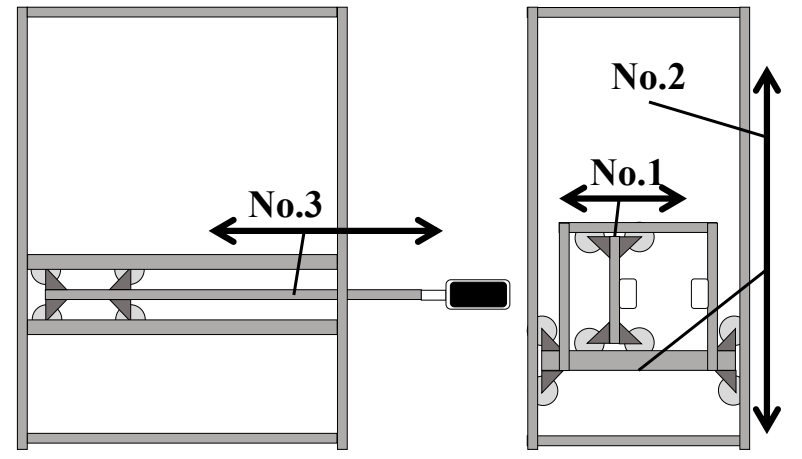

Figure 1: Robot over view.

rooms", a collection of reports by the Architectural Institute of Japan in 1966, the dust generation rate when two people are in one room is $2.44 \times 10^{-3}\left(\mathrm{~m}^{3} / \mathrm{s}\right)$ [12].

Many people have pets. According to the national survey of the Pet Food Association's in 2020, 6.8 million households have 8.49 million dogs and 5.51 million households have 9.64 million cats [13]. Dogs and cats shed a lot of hair. One Labrador retriever sheds 238 hairs per 30 minutes, which is 11424 hairs a day [14]. Many pet owners are troubled by their pets'hair loss [15] [16] Kärcher Japan Co. surveyed 500 men and women who own a dog or cat in 2019 [17]. According to it, the biggest concern for the majority of them when cleaning was dirt around the floor caused by pet hair. Animal hair, which falls on the floor in large quantities, adheres to our clothes as we sit or walk. In this way, if you keep animals such as dogs and cats indoors, a lot of animal hair will stick to your clothes in addition to dust. 


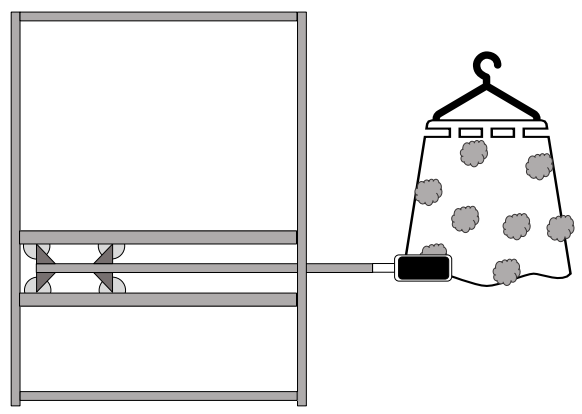

(a) Start

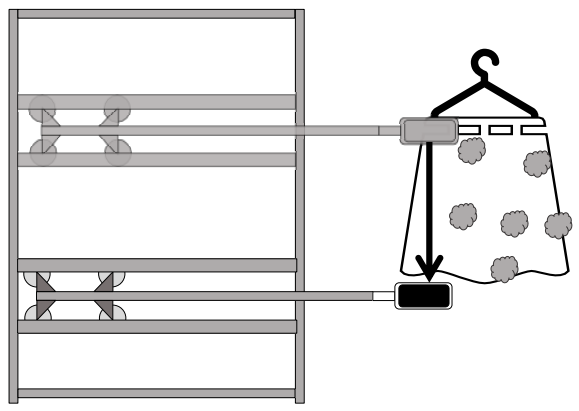

(c) Step 2

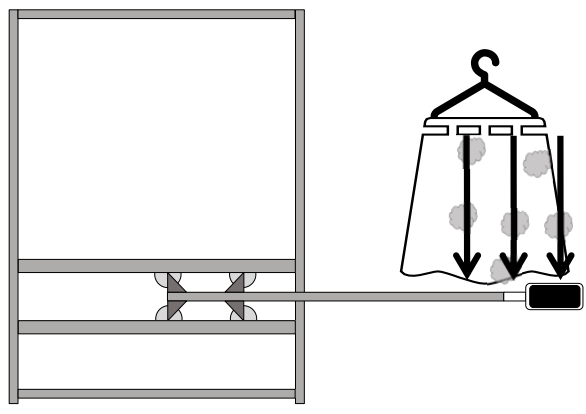

(e) Step 4

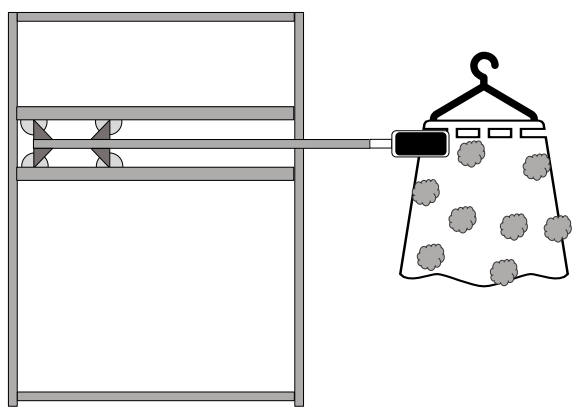

(b) Step 1

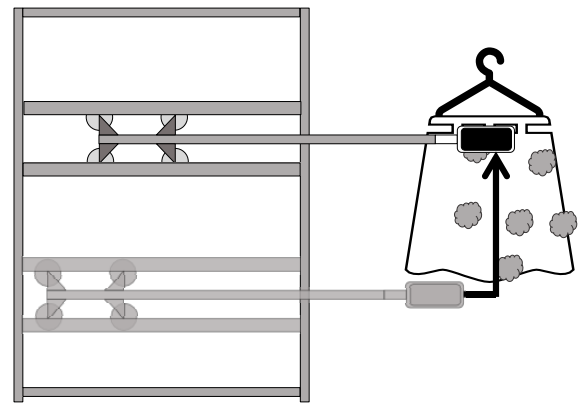

(d) Step 3

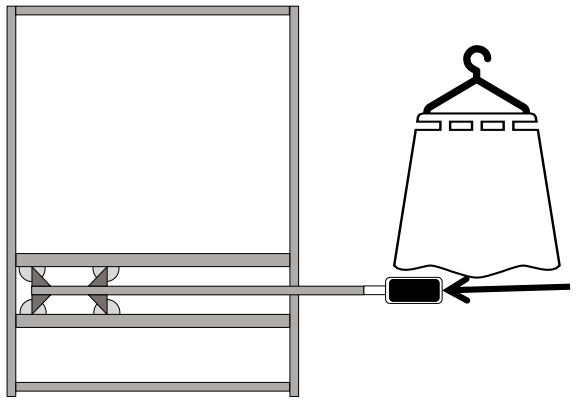

(f) Step 5

Figure 2: Operating procedure.

Dust and animal hair on clothes are mostly removed by using rolls of adhesive tape, special brushes, and other useful items. However, these need to be done manually, which can be a big burden if you have a lot of clothes or if you have a dog or cat.

Many people spend a lot of time grooming themselves in the morning before going to work [18]. And many people feel that they don't have time in the morning. According to the survey on how people spend their mornings on workdays conducted in 2012 by Lion Corporation shows, $65.1 \%$ of 1000 office worker aged 20-50 answered that don't have enough time on the morning of the day that have to go to work [19]. In addition, in this survey, when asked how much they could afford to pay for a minute of their busy morning, the average answer was 531 yen.

In this paper, we propose a robot automatically to remove dust from clothes to reduce the burden at home. This robot can automatically remove dust from both sides of clothes by holding the clothes on the hanger between two dust remov- ing brushes and moving them from the top to the bottom. In addition, the brushes can be extended sideways to remove dust from the entire clothes. By using this robot, you can save time and effort by removing dusts.

\section{Automatic Dust Catcher Robot of Clothes}

This robot can hold clothes between two brushes and move them down to remove dust from the clothes. In addition, by extending the brush and repeating these motions, it can remove dust from the entire clothes. As shown in Fig. 1, each moving part corresponds to the following axis, No.1 for holding the clothes between the brushes, No.2 for moving the brushes up and down to remove dust from the clothes, and No.3 for extending the brushes to remove dust from the whole clothes. The moving parts move in the direction of the arrows, respectively.

The user hangs the clothes at a predetermined position using a hanger rack. The predetermined position is where the clothes are placed between the brushes of the robot. Press 


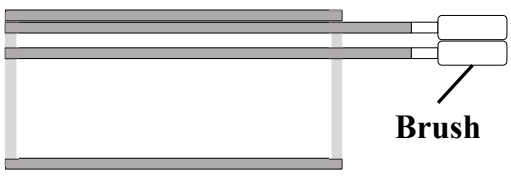

(a) Start

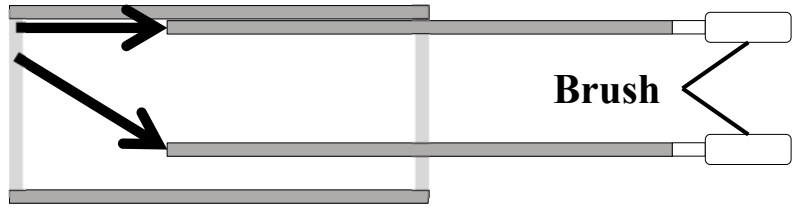

(b) In motion

Figure 3: Opening and stretching motion.

the start button to start the robot's operation. Once started, the robot repeatedly moves its three axes to brush the entire clothes and remove dust from the clothes. When the robot finishes dusting the entire clothes, it shrinks the brushes to finish the operation.

The above procedure is explained concretely with illustrations. First, set the clothes as shown in Fig. 2(a). When the operation starts, the robot brings the brush to the top of the clothes as shown in Fig. 2(b). When the brush reaches the top of the clothes as shown in Fig. 2(c), the robot pinches the clothes between the brushes and lowers the brush down to remove dust from the clothes. Then, as shown in Fig. 2(d), the motion of releasing the brush from the clothes and stretching the brush sideways are performed simultaneously, followed by the motion of raising the brush. Figure 3 shows the brushes are releasing from the clothes and the brushes are extending at the same time. As shown in Fig. 2(e) and Fig. 2(f), the above operation is repeated, and when the dust is removed from the entire clothes, the brush is shrunk to finish the operation.

The mechanism of each axis is shown in the sections of 2.1 2.3.

2.1 Axis to hold clothes In order to remove dust from clothes, it is necessary to keep the brush close to the clothes and to hold the clothes in place. In order to remove dust from clothes in this study, we adhere and fix by sandwiching the clothes between the two brushes. The mechanism to perform the above operation is explained in the following illustrations. As shown in Fig. 4(a), a motor and a wire are attached to move the shaft left and right in order to pinch the clothes. Furthermore, a sensor is used to control the state of the axis as shown in Fig. 4(b).

2.2 Axis to move the brush up and down The brush needs to be moved up and down in order to collect the dust from the clothes. The mechanism for moving the shaft up and down is explained in the following illustrations. To move the shaft up and down, attach the motor and the wires as shown in Fig. 5(a). Furthermore, a sensor is attached to control the state of the axis as shown in Fig. 5(b).

2.3 Axis to extend the brush The shoulder width of the clothes is longer than the length of the brush. Thus, to
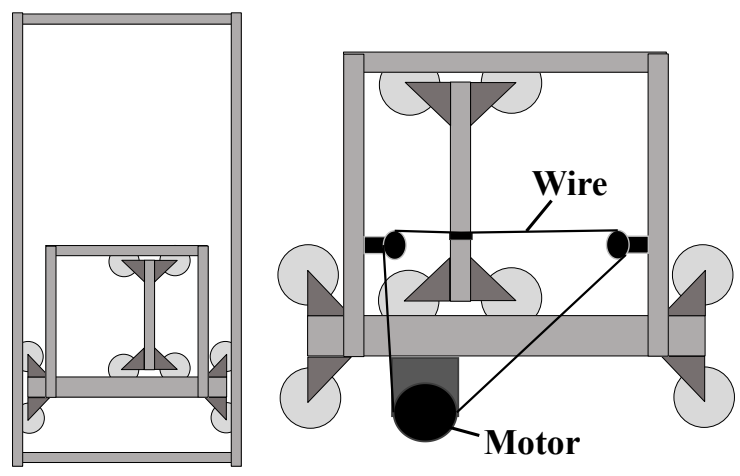

(a) Wire and motor
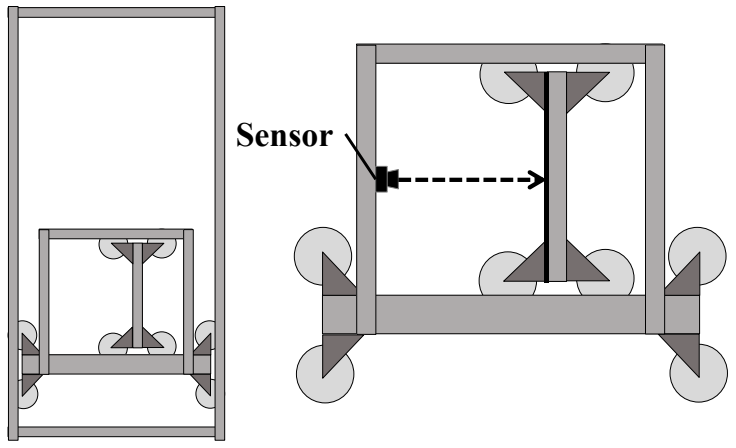

(b) Sensor

Figure 4: Hold clothes.

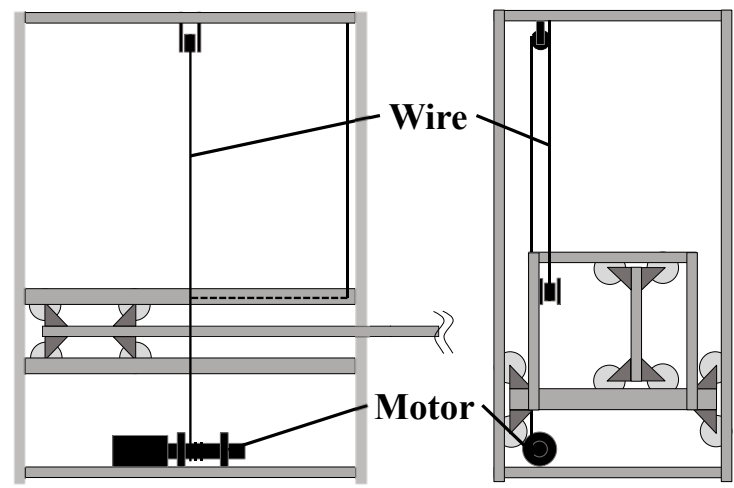

(a) Wire and motor

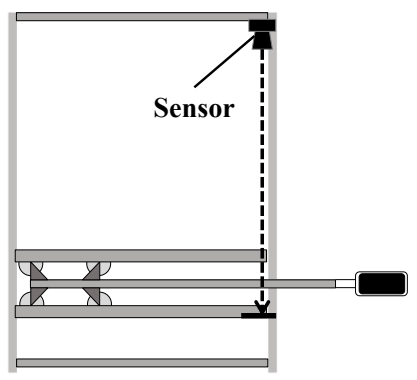

(b) Sensor

Figure 5: Moves up and down.

get the dust off the entire clothes, need to brush it several time. In this paper, we realize remove dust from the entire clothes by stretching the brush several times. The mecha- 


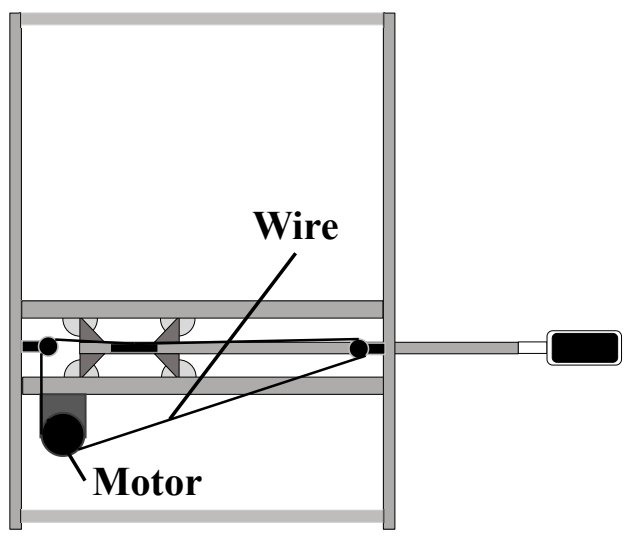

(a) Wire and motor

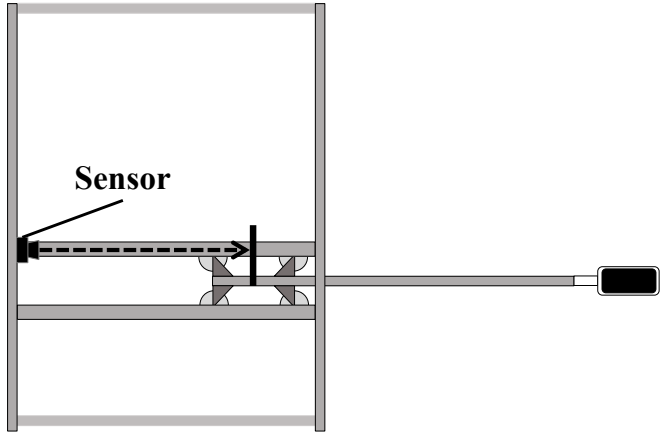

(b) Sensor

Figure 6: Extend.

nism to perform the above operation is explained using the next illustrations. To extend the shaft, attach the motor and the wires as shown in Fig. 6(a). In addition, attach the sensor shown in Fig. 6(b) to control the state of the axis.

\section{Robot of prototype}

In this section, we describe the prototype of the robot described in Chapter 2. The overall view of the robot is shown in Fig. 7. As shown in Fig. 7, the robot is box-shaped, but the arm with a brush (Copa Corporation, MUGENBRUSH) sticks out to pinch clothes. The size of the box part of this robot is about $740 \mathrm{~W} \times 495 \mathrm{D} \times 1000 \mathrm{H}(\mathrm{mm})$. The length of the protruding arm is $380 \sim 830(\mathrm{~mm})$. Put the hanger rack so that the clothes come to the position shown in Fig. 2(a). The robot automatically removes dust from clothes hung on the hanger rack.

The details of the three-axis described in Chapter 2 are explained in the sections of 3.1 3.3.

3.1 Axis to hold clothes A photo of the axis to hold clothes is shown in Fig. 8. As shown in Fig. 8, only one of the brushes has a drive unit on each side. The drive units are called the drive unit 1 in Fig. 8(a) and the drive unit 2 in Fig. 8(b), respectively. These drive units are driven simultaneously to pinch the clothes. The brushes are driven up to $160 \mathrm{~mm}$ in the pinching direction. This motion is achieved by winding the wire using a motor (TAMIYA, 4Speed Worm Gearbox H.E.) and pulleys. The motor is run using a $5(\mathrm{~V})$ DC power supply, and the drive direction is

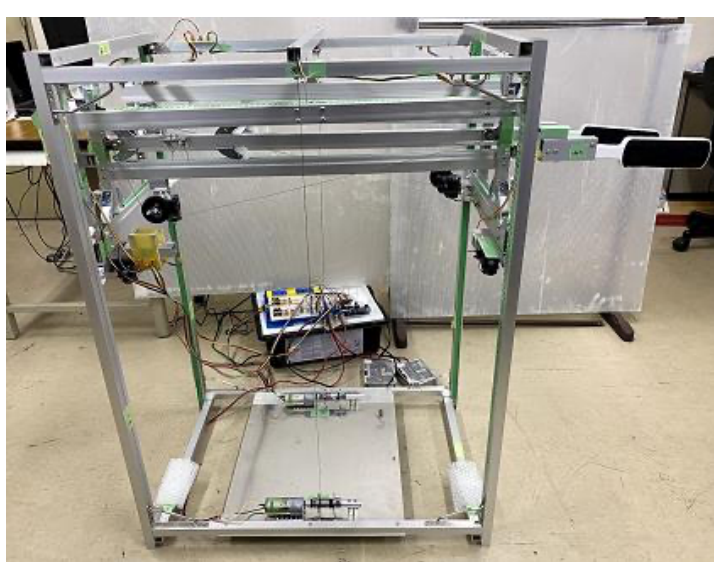

(a) Front view

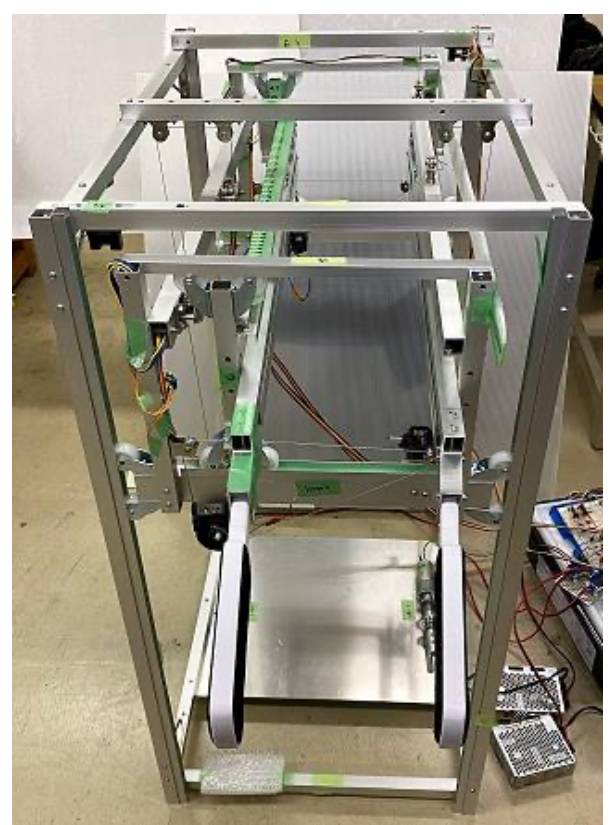

(b) Side view

Figure 7: Extend.

controlled using a motor driver (Toshiba, TA7291P). In addition, a distance sensor (Sharp, GP2Y0E03) is installed in each of the two driving sections, and the width of the pinching is controlled using an arduino.

3.2 Axis to move the brush up and down Figure 9 shows the mechanism for moving the brush up and down in the prototype. The two brushes are moved up and down by simultaneously driving the two mechanism for extending brushes as shown Fig. 9. Figure 10 shows the top view of the arms with the brushes attached. Each arm is called Arm 1 and Arm 2, as shown in Fig. 10. The brushes are driven maximum of $520(\mathrm{~mm})$ in width of up and down. The motor (TAMIYA, AO-8037 Geared Motor 540K300) and the wires are used for this movement. The motor runs on a $12(\mathrm{~V})$ DC power supply and the wire is wrapped around the pipe. The motor driver (Pololu, VNH3SP30) is used to control the drive direction. In addition, a distance sensor (Sharp, GP2Y0A02) is installed in each of the two drive sections, and the height at which it is moved can be controlled using 


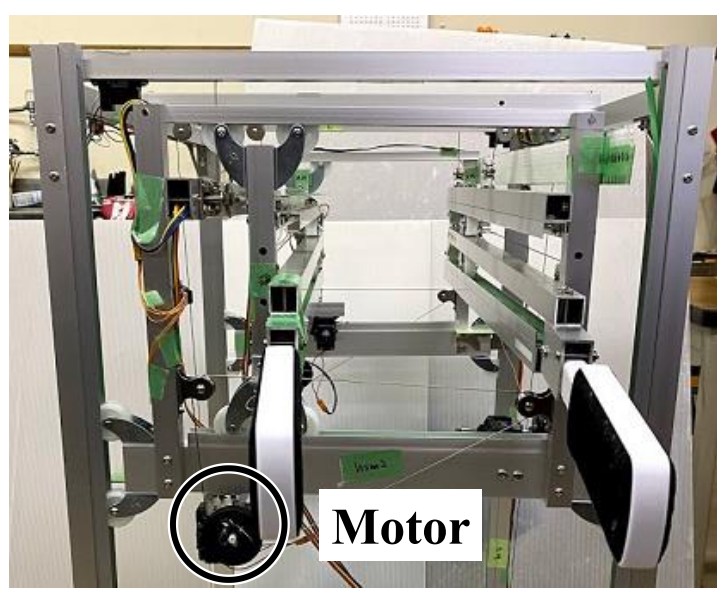

(a) Drive unit 1

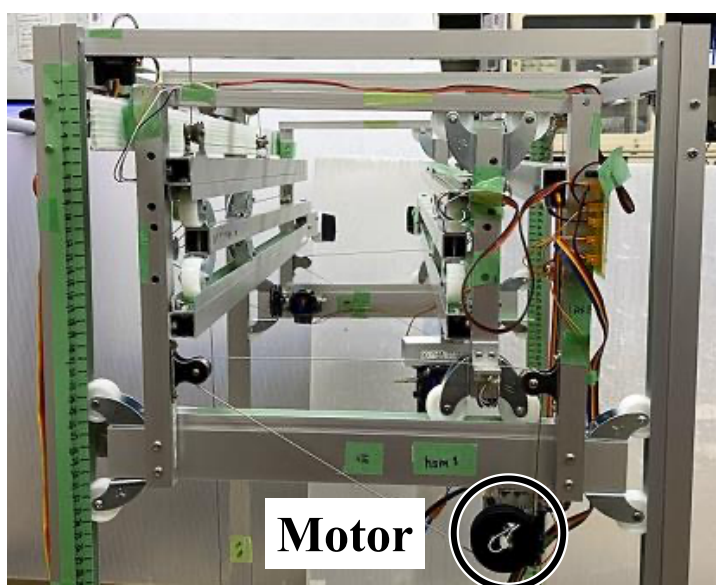

(b) Drive unit 2

Figure 8: Drive units.

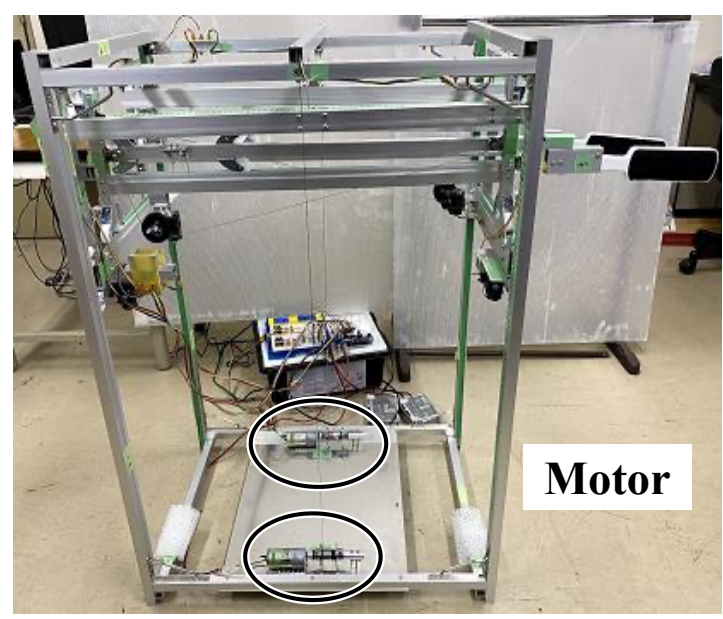

Figure 9: Moves up and down.

an arduino.

3.3 Axis to extend the brush Figure 11 shows the mechanism for extending the brushes of the prototype as in Section 3.2, we distinguish the arms to be driven as in Fig. 10. The brushes are driven the maximum of $450(\mathrm{~mm})$. This movement is achieved by using a motor (TAMIYA, 4Speed Worm Gearbox H.E.) and a pulley to wind the wire.

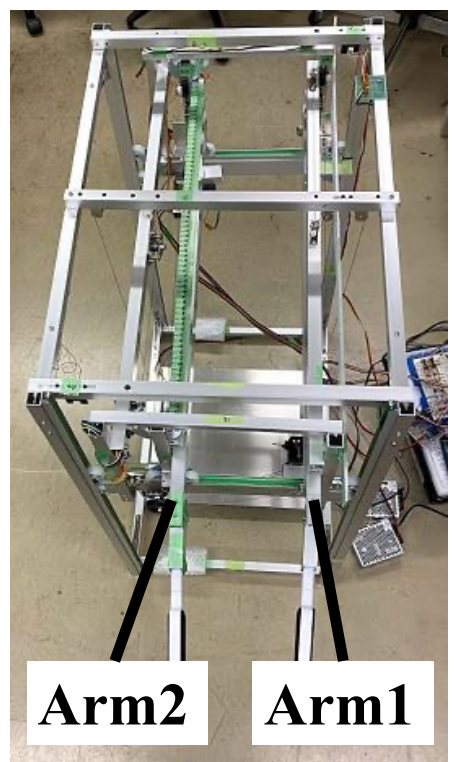

Figure 10: Arm.

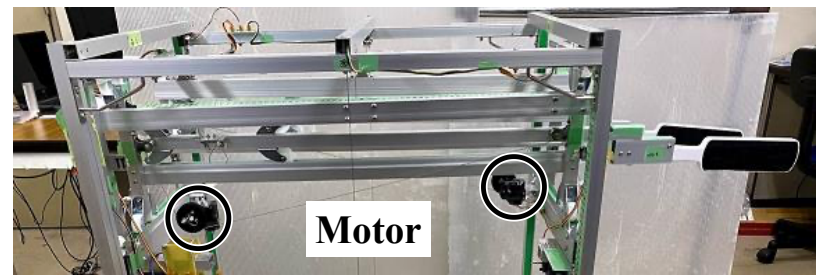

Figure 11: Extends.

The motor is run using a $5(\mathrm{~V})$ DC power supply, and the drive direction is controlled using a motor driver (Toshiba, TA7291P). In addition, the axis to be extended can be seen by the distance sensor (Sharp, GP2Y0A21YK0F), and the distance to extend the brush can be controlled using the arduino.

\section{Experiment}

In this paper, the entire clothes are dusted repeatedly by controlling three axes: the axis that holds the clothes, the axis that moves the brush up and down, and the axis that extends the brush. After checking the operation of each axis, we checked the operation of the entire system. We ran the robot ten times to see if it could remove the dust from the clothes. The outputs of all the sensors used in the prototype were averaged 50 times to reduce an error.

The experiments conducted in this study are shown in the sections of $4.1 \sim 4.5$.

4.1 Axis to hold clothes We checked the output of the sensor and determined the threshold value to control each axis. We checked the value of the output voltage of the sensor when the clothes were pinched and when it was open. Table 1 shows the outputs when the arm was open, and when it was closed. The values in Table 1 were used as threshold values to control the axis. When both drives had not reached the threshold, both drives were moved simultaneously. When only one of them reached the threshold, only the drive unit that had not reached the threshold was 


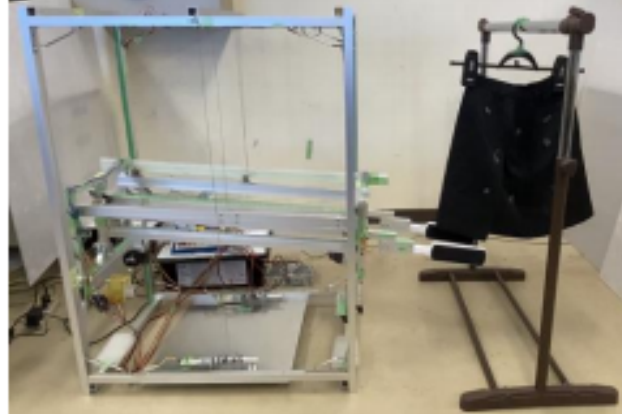

(a) Start

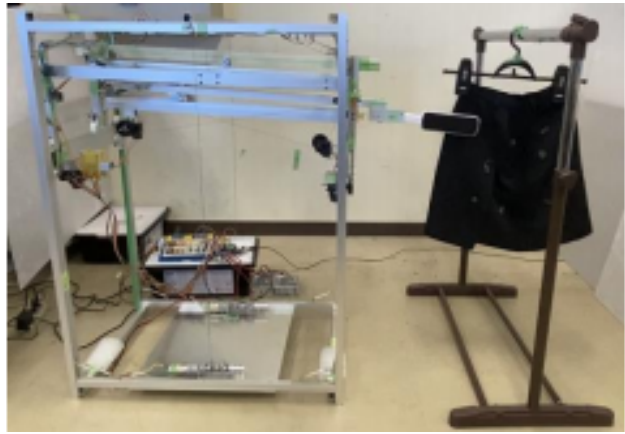

(c) Step 3

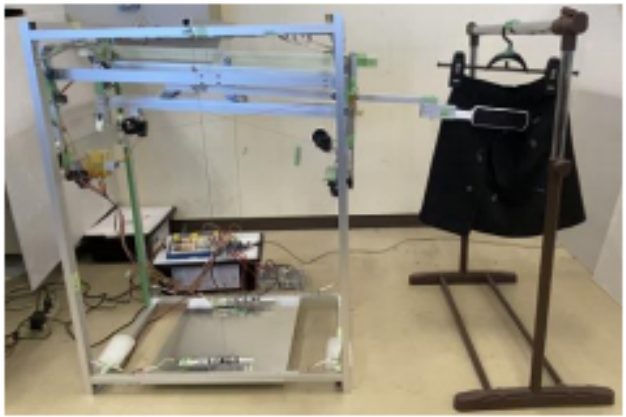

(e) Step 4

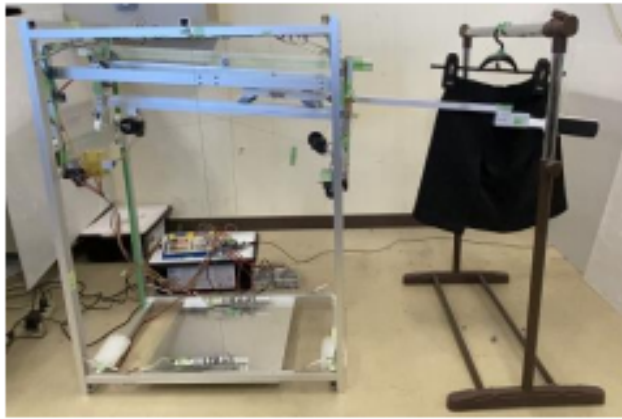

(g) Step 6

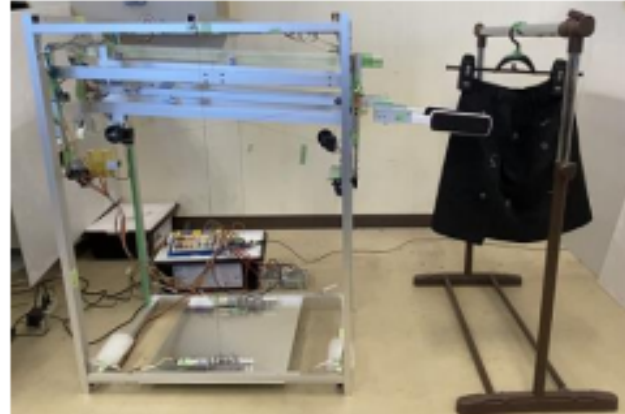

(b) Step 2

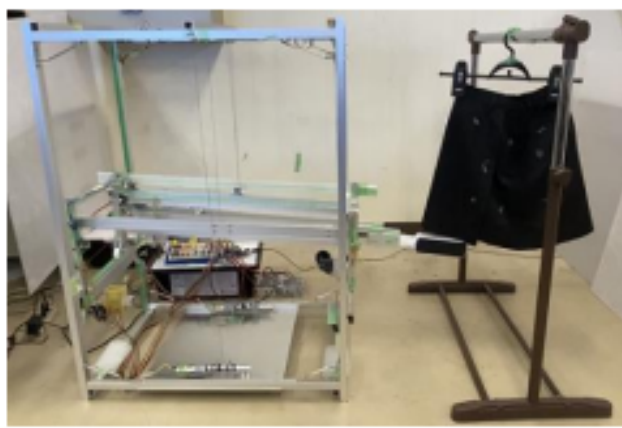

(d) Step 4

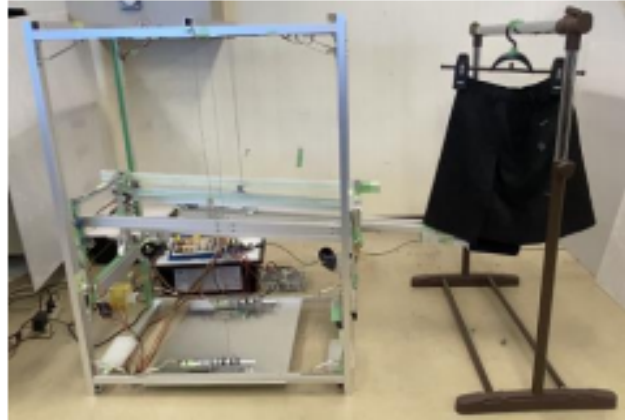

(f) Step 5

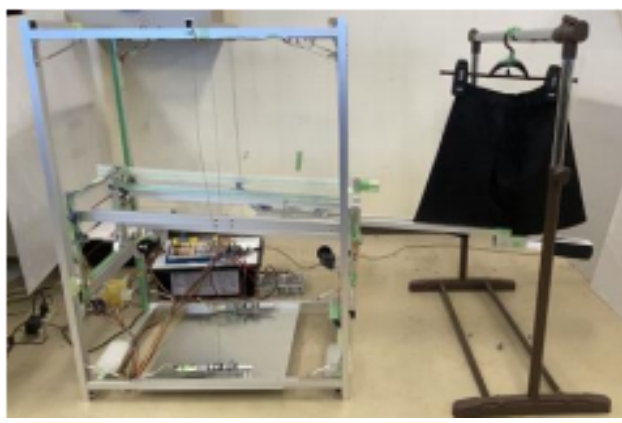

(h) Step 7

Figure 12: Experiment.

Table 1: Hold clothes.

\begin{tabular}{ccc}
\hline \multirow{2}{*}{ Position } & \multicolumn{2}{c}{ Output voltage(V) } \\
& Drive1 & Drive2 \\
\hline open & 2.13 & 2.09 \\
close & 1.42 & 1.45 \\
\hline
\end{tabular}

moved.
4.2 Axis to move the brush up and down In this experiment, a hanger rack of $840 \mathrm{~W} \times 1000 \mathrm{H}(\mathrm{mm})$ and a hanger of $400 \mathrm{~W} \times 210 \mathrm{H}(\mathrm{mm})$ was used. If clothes are placed on a hanger, the top edge of the clothes are assumed to be directly under the hanger. When I put the hangers on the hangers, the top of the clothes was $800(\mathrm{~mm})$ above the ground. We assumed that the drive part needed to be moved within the range of $300-800 \mathrm{~mm}$ from the ground in order 
Table 2: Moves up and down.

\begin{tabular}{ccc}
\hline \multirow{2}{*}{ Position } & \multicolumn{2}{c}{ Output voltage(V) } \\
& arm1 & arm2 \\
\hline Topmost & 2.25 & 1.71 \\
Lowermost & 1.05 & 0.83 \\
\hline
\end{tabular}

Table 3: Extend.

\begin{tabular}{ccccc}
\hline \multirow{2}{*}{ Position } & \multicolumn{2}{c}{ Distance(mm) } & \multicolumn{2}{c}{ Output voltage(V) } \\
& arm1 & arm2 & arm1 & arm2 \\
\hline Initial & 140 & 110 & 1.86 & 2.15 \\
1 & 260 & 230 & 1.16 & 1.12 \\
2 & 380 & 350 & 0.87 & 0.74 \\
3 & 500 & 470 & 0.76 & 0.54 \\
\hline
\end{tabular}

to remove dust from the top to the bottom of the clothes. We checked each output voltage when the arm was at the top and at the bottom. The output voltages when the arm is at the top and at the bottom are shown in Table 2. The two-arms ware moved up and down using the values in Table 2 as threshold values. When both arms had not reached the threshold, both arms were moved simultaneously. When only one of them reached the threshold, only the drive unit of the one that had not reached the threshold was moved.

4.3 Axis to extend the brush The width of the brush is $150(\mathrm{~mm})$ and the width of the hanger is $400(\mathrm{~mm})$ from the section 4.2. To remove dust from the entire clothes, the arm was extended every $120(\mathrm{~mm})$ to remove the dust. Table 3 shows the position of each arm and the output voltage when moved every 120 (mm). Using the values in Table 3 , the arm was moved from the initial position to position 3. After the arm reached position 3 , it was returned to the initial position.

In this paper, in order to speed up the operation, we performed the opening operation and extending operation of the brush simultaneously.

4.4 Check the entire system An experiment was conducted to remove dust from clothes hanging on a hanger rack using a prototype robot. A $100(\%)$ polyester, small size short pants was used as a sample of the clothes. The hangers were fixed to the hanger rack with curing tape to prevent shaking. Dust was collected and evenly adhered to the short pants. The experiment was conducted after hanging the short pants on a hanger rack so that they were between the brushes as shown in Fig. 2(a).

The operation of the prototype during the experiment is shown in Fig. 12. First, the arms were opened and raised to the top of the short pants as shown in Fig. 12(a) and Fig. 12(b). Next, as shown in Fig. 12(c) and Fig. 12(d), the short pants were pinched and the arm was lowered to remove dust. Then, after extending the arm while opening it, the dust was removed from the short pants as shown in Fig. 12(e) and Fig. 12(f). As shown in Fig. 12(g) and Fig. 12(h), by repeating the dust removal operation a total of four times while extending the arm, the robot removed dust from the entire clothes. After the entire short pants

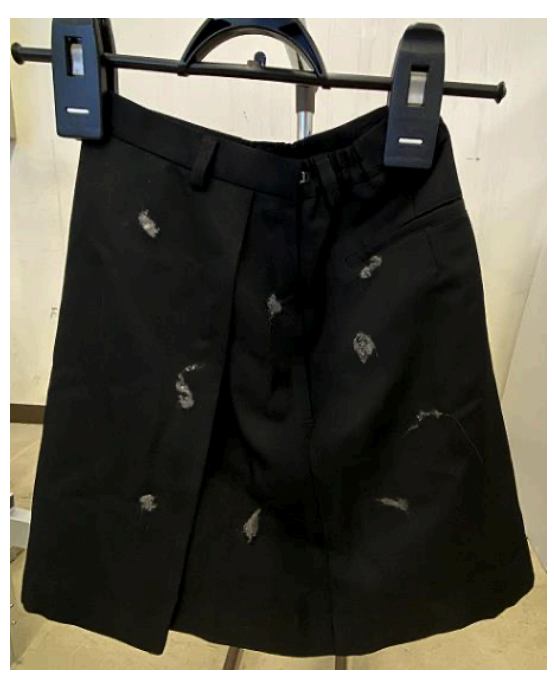

(a) Before

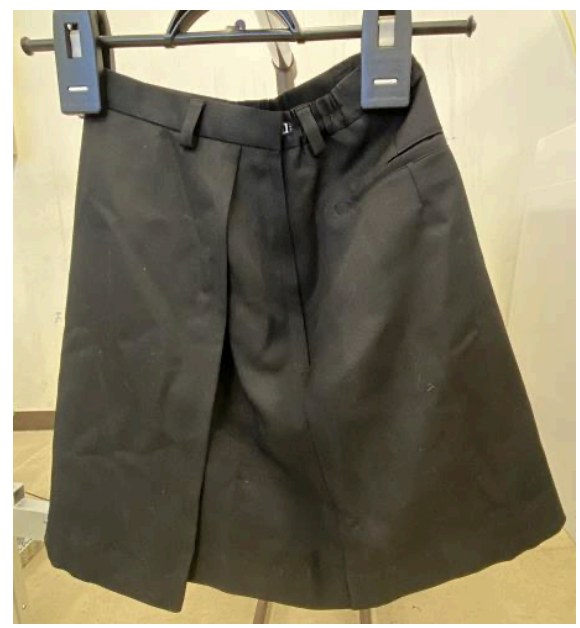

(b) After

Figure 13: Result.

were removed dusts, the arm was retracted while opening the arm.

As a result of the experiment, the operating time of the prototype was about 2 minutes. Figure 13 shows the short pants before and after the robot removed the dust. From Fig. 13, it was confirmed that the dust on the clothes was removed.

4.5 Checking the robot's performance We performed the experiment described in section 4.4 ten times and visually checked whether the robot could remove dust from the clothes or not. The results of four of the ten experiments are shown in Fig. 14 through Fig. 17. As shown in Fig. 14 Fig. 17, most of the dust on the clothes was removed in the experiment. However, in Experiment B, the dust remained on the clothes after the experiment, as shown in Fig. 15(d). In only experiment B out of the ten experiments, it was confirmed that the dust remained on the clothes after the experiment. 


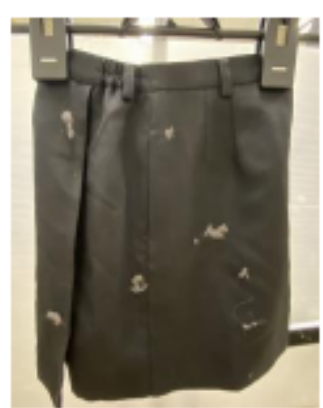

(a) Front side before test

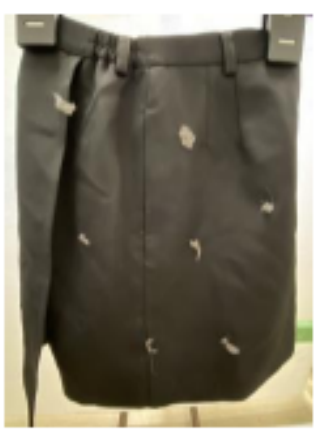

(a) Front side before test

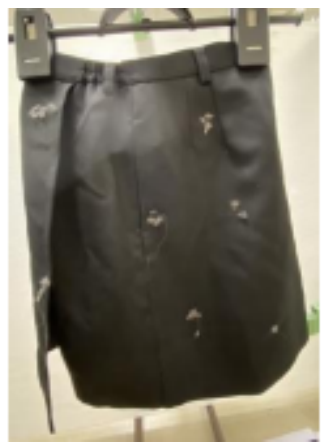

(a) Front side before test

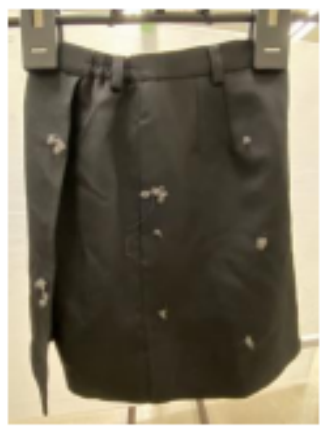

(a) Front side before test

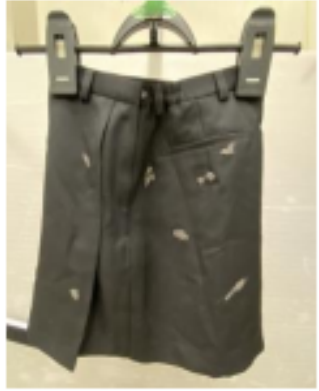

(b) Back side before test

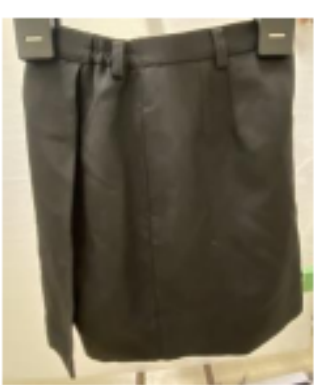

(c) Front side after test

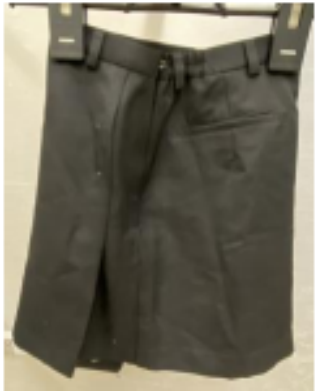

(d) Back side after test

Figure 14: Experiment A.

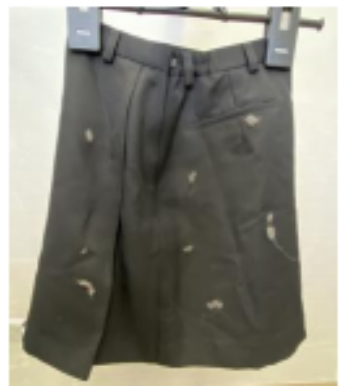

(b) Back side before test

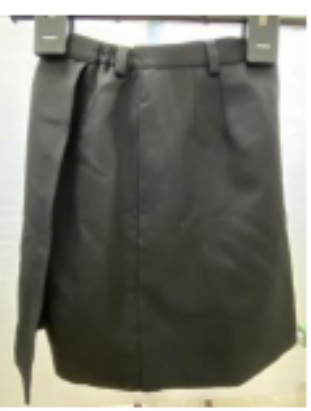

(c) Front side after test

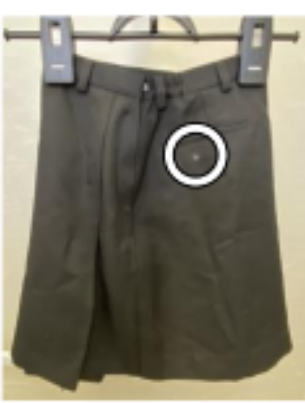

(d) Back side after test

Figure 15: Experiment B.

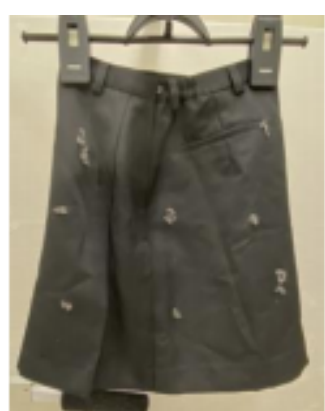

(b) Back side before test

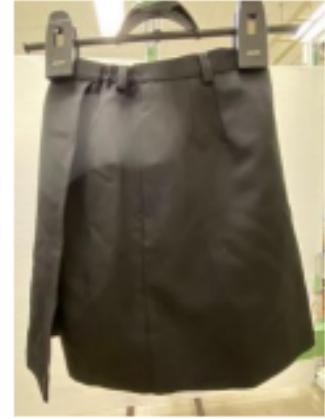

(c) Front side after test

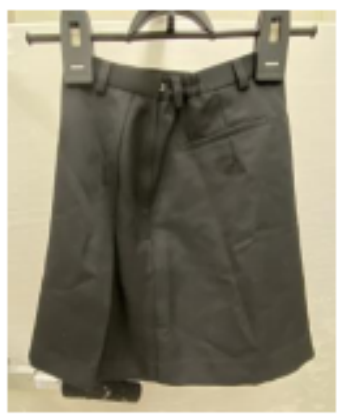

(d) Back side after test

Figure 16: Experiment C.

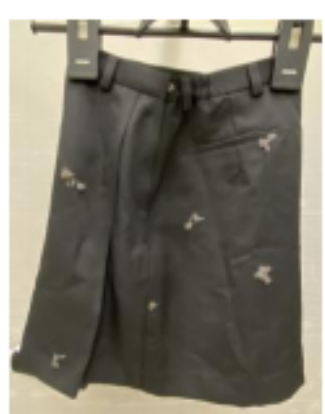

(b) Back side before test

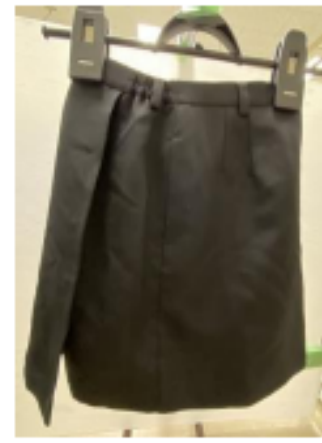

(c) Front side after test

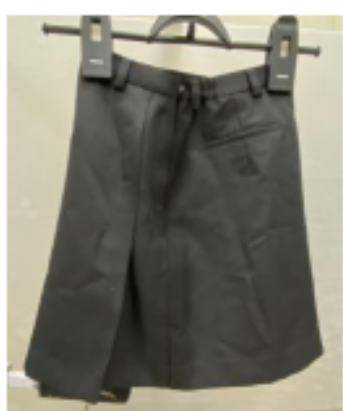

(d) Back side after test

Figure 17: Experiment D.

\section{Conclusion}

In this study, we developed a robot that removes dust from clothes on hangers by controlling three axes: the axis that holds the clothes, the axis that moves the brush up and down, and the axis that extends the brush. As a result of the experiment, we were able to remove most of the dust 
from the clothes. This robot can automatically remove dust by user pressing the start button. The user only needs to hang the clothes on the hanger rack before robot's work and collect the clothes after the it's work. The user of the robot no longer needs to use his or her hands and time for dusting clothes, and can make better use of them for other things. Therefore, it is now possible to reduce the labor and time consumed in removing dusts of clothes.

In the future, we plan to make improvements to this robot. We would like to make it possible for robots to operate automatically and continuously on multiple pieces of clothes. Also, the brush used for this robot comes with its own case. When this brush is plunged into the case and pulled out, the dust on the brush is transferred to the case and accumulates. We are planning to attach this case to a robot to automatically remove the dust from brushes.

\section{References}

[1] Y. Tuduki and M. Narita, "Twin cases of anaphylaxis caused by ticks in okonomiyaki flour", Journal of the Japanese Society of Internal Medicine, Vol.104, No.5, pp.986-990, 2015.

[2] LSI Medience Corporation, Allergy Information, https:// www.medience.co.jp/allergy/koumoku.html, access date: 2021.03 .12

[3] I. Suzuki, F. Okada, K. Uchimura, K. Ito, T. Mitsubayashi, T. Akasaka and K. Maeda, "DIAGNOSIS AND TREATMENT OF CAT ALLERGY AND DOG ALLERGY IN ASTHMATIC CHILDREN", The Japanese Journal of Pediatric Allergy and Clinical Immunology, Vol.1, No.1, pp.25-31, 1987.

[4] S. Seno, T. Enomoto, Y. Dake, Y. Saito, H. Ikeda, H. Funakoshi, H. Sogo, A. Shibano, T. Sakota, M. Suzuki and Y. Yazawa, "Epidemic Study of Pet Allergy in Wakayama Prefecture", Nippon Jibiinkoka Gakkai Kaiho, Vol.106, No.7, pp.750-753, 2003. DOI: 10.3950/jibiinkoka.106.750

[5] M. Nambu, "Indoor Allergen Control in Allergic Children", Journal of the Japanese Society of Pediatric Allergy, Vol.24, No.2, pp.203-216, 2010.

[6] R. Muko, H. Matsuda and A. Tanaka, "Animal model of skin allergy - Screening and evaluation of functional substances”, Functional Food Research, Vol.14, pp.23-29, 2018. DOI: $10.32153 / \mathrm{ffr} .14 .023$

[7] A. Akasawa, "Can an Allergic Child Have a Pet? From a Pro's Perspective", Journal of the Japanese Society of Pediatric Allergy, Vol.28, No.1, pp.58-65, 2014.

[8] K. Abe, "Literature Guide about Social Attitude in Immersive Virtual Environment", Cognitive Science, Vol.26, No.4, pp.509-515, 2019. DOI: 10.11225/jcss.26.509

[9] Tameny, What I changed for marriage activity: Clothes are ranked first for both men and women, Vol. 146, https:// www.p-a.jp/research/report_146.html, access date: 2021.03.12

[10] Tameny, The number one requirement for a marriage partner is someone with a sense of cleanliness, Vol. 144, https:// www.p-a.jp/research/report_144.html, access date: 2021.03.12

[11] Statistics Bureau, Ministry of Finance, Prefecture, Number of general households by number of persons in household and number of persons in household by type of household, https://www.stat.go.jp/data/nihon/02 .html, access date: 2021.03 .12

[12] F. Syouichi, K. Keiichi, K. Kikuji and Y. Susumu, "3013 The amount of dust generated indoors", Proceedings of the Architectural Institute of Japan, Extra Issue, Extra Edition, Academic Abstracts, Vol.41, pp.395-, 1966.

[13] Pet Food Association of Japan, National Survey of Dog and Cat Ownership - Key Indicators and Summary -, https: //petfood.or.jp/data/chart2020/3.pdf, access date: 2021.03.12

[14] Y. Masaru, "Hair Loss in Labrador Retriever", Japan Assistance Dog Science Research, Vol.2, No.1, pp.36-40, 2008.

[15] Iris Ohyama, Cat Census in 2019, https://www. irisplaza.co.jp/media/A14025807205, access date: 2021.03.12

[16] au insurance Co., Survey on Pet Health, , https://www . au@sonpo.co.jp/corporate/news/detail-208.html, access date: 2021.04 .22

[17] Kärcher Japan Co., Survey on Cleaning and Pets, https://prtimes.jp/main/html/rd/p/000000010. 000022995.html, access date: 2021.03.12

[18] Do House Inc., Survey results on morning activities, https: //www. dohouse.co.jp/news/research/20150421/, access date: 2021.04 .22

[19] Lion Co., Survey on how people spend their mornings on workdays, https://www.lion.co.jp/ja/company/ press/2012/802, access date: 2021.04.22

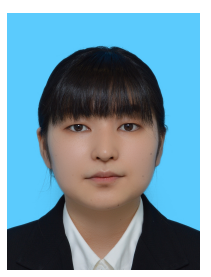

Natsuki Nakamoto (Non-member) was born in Fukuoka, Japan. She is a student in Department of Creative Engineering of National Institute of Technology, Kitakyushu College. Her current research interest is Sensing System.

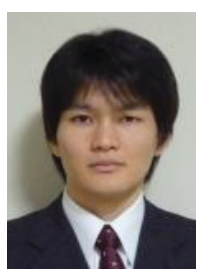

Yhuki Kitazono (Member) was born in Kumamoto, Japan, on December, 1984. He received the B.S., M.S., and Ph.D. degrees in Electrical Engineering from Kyushu Institute of Technology in 2007, 2009, and 2011. From 2011 to 2015, he was an Assistant Professor at the Kitakyushu National College of Technology. Since 2015, he has been an Associate Professor at the National Institute of Technology, Kitakyushu College. His current research interests include measurement, sensors, and robotics. He is a member of IIAE. 